\title{
Equilibrium Outcomes in Repeated Two-Person, Zero-Sum Games
}

\author{
Guilherme Carmona \\ Universidade Nova de Lisboa
}

October 15,2002

\begin{abstract}
We will consider repeated two-person, zero-sum games in which the preferences in the repeated game depend on the stage-game preferences, although not necessarily in a time-consistent way. We will assume that each player's repeated game payoff function at each period of time is strictly increasing on the stage game payoffs and that the repeated game is itself a zero-sum game in every period. Under these assumptions, we will show that an outcome is a subgame perfect outcome if and only if its components are all Nash equilibria of the stage game.
\end{abstract}

\section{Introduction}

Recently, there has been an increasing interest in time-inconsistent, and its consequences for economic theory, and policy. Much of this interest was motivated by the work of Laibson [4], Jovanovic and Stolyarov [2], and Kocherlakota [3], which have shown that time-inconsistent preferences can change a model's implications for economic policy. In contrast, we will show that in a two-person, zero-sum game time-inconsistent preferences have no effect over the equilibrium outcomes that can arise, and so have no effect over the model's implication for economic policy.

Our main result can be stated as follows: consider a repeated two-person, zero-sum games in which the preferences in the repeated game depend on the stage-game preferences, although not necessarily in a time-consistent way. 
Assume that each player's repeated game payoff function at each period of time is strictly increasing on the stage game payoffs, and, if the repeated game is itself a zero-sum game in every period. Under these assumptions, we will show that an outcome is a subgame perfect outcome if and only if all its components are all Nash equilibria of the stage game.

Given the recent interest on the economic effects of time-inconsistent preferences, it is interesting to know what game-theoretic results change by assuming time-inconsistent preferences. This question seems natural to us since, as Peleg and Yaari [5] and Goldman [1] pointed out, the appropriate way of modelling time-inconsistency in preferences is through the concept of subgame perfect equilibrium of a game between an agent and his future selves. ${ }^{1}$ Therefore, it seems necessary to know how game-theoretic results will change in order to understand the economic effects of time-inconsistent preferences.

It should be noted that the conclusion of our main result belongs to the oral tradition of game theory, at least when the repeated game payoffs are given by the discounted sum of stage game payoffs. The contribution of our work is to provide a simple proof of that result, and to show that it holds under quite general assumptions.

\section{Notation and definitions}

A two-person, zero-sum game $G$ is defined by

$$
G=\left(A_{1}, A_{2}, u_{1}, u_{2}\right),
$$

where for all $i=1,2$ : (1) $A_{i}$ is a finite set of player $i$ ' actions, and (2) $u_{i}: A \rightarrow \mathbb{R}$, where $A=A_{1} \times A_{2}$, is player $i$ ' payoff function; the player's payoff functions satisfy

$$
u_{1}(a)+u_{2}(a)=0,
$$

for all $a \in A$. Let $S_{i}=\Delta\left(A_{i}\right), S=S_{1} \times S_{2}$, and $u_{i}: S \rightarrow \mathbb{R}$ be the usual extension to mixed strategies.

Let, for $i=1,2, v_{i}=\min _{s_{-i}} \max _{s_{i}} u_{i}\left(s_{i}, s_{-i}\right)$, and $N E=\{s \in S$ : for all $i=1,2, u_{i}(s) \geq u_{i}\left(\tilde{s}_{i}, s_{-i}\right)$, for all $\left.\tilde{s}_{i} \in S_{i}\right\}$. The set $N E$ is the set of Nash equilibria of $G$, and $v_{i}$ is the minmax level for player $i$.

\footnotetext{
${ }^{1}$ The concept of time-inconsistent preferences was itself introduced by Strotz [6].
} 
The supergame of $G$ consists of an infinite sequence of repetitions of $G$ taking place in periods $t=1,2,3, \ldots$ At period $t$ the players make simultaneous moves denoted by $s_{i}^{t} \in S_{i}$ and then each player learn his opponent's move.

For $k \geq 1$, a $k$-stage history is a $k$-length sequence $h_{k}=\left(s^{1}, \ldots, s^{k}\right)$, where, for all $1 \leq t \leq k, s^{t} \in S$; the space of all $k$-stage histories is $H_{k}$, i.e., $H_{k}=S^{k}$ (the $k$-fold Cartesian product of $S$.) The notation $e$ stands for the unique 0-stage history - it is a 0-length history that represents the beginning of the supergame. The set of all histories is defined by $H=$ $\bigcup_{n=0}^{\infty} H_{n}$.

For every $h \in H$, define $h^{r} \in S$ to be the projection of $h$ onto its $r^{\text {th }}$ coordinate. For every $h \in H$ we let $\ell(h)$ denote the length of $h$. For two positive length histories $h$ and $\bar{h}$ in $H$ we define the concatenation of $h$ and $\bar{h}$, in that order, to be the history $(h \cdot \bar{h})$ of length $\ell(h)+\ell(\bar{h}):(h \cdot \bar{h})=$ $\left(h^{1}, h^{2}, \ldots, h^{\ell(h)}, \bar{h}^{1}, \bar{h}^{2}, \ldots, \bar{h}^{\ell(\bar{h})}\right)$. We also make the convention that $e \cdot h=$ $h \cdot e=h$ for every $h \in H$.

It is assumed that at stage $k$ each player knows $h_{k}$, that is each player knows the actions that were played in all previous stages. Regarding strategies, players chose behavioral strategies, that is, in each stage $k$, they choose a function from $H_{k-1}$ to $S_{i}$ denoted $f_{k}^{i}$, for player $i=1,2$. The set of player $i$ 's strategies is denoted by $F_{i}$, and $F=F_{1} \times F_{2}$ is the joint strategy space. Finally, a strategy vector is $f=\left(\left\{f_{k}^{i}\right\}_{k=1}^{\infty}\right)_{i=1,2}$.

Given an individual strategy $f_{i} \in \Sigma_{i}$ and a history $h \in H$ we denote the individual strategy induced by $f_{i}$ at $h$ by $f_{i} \mid h$. This strategy is defined pointwise on $H$ : $\left(f_{i} \mid h\right)(\bar{h})=f_{i}(h \cdot \bar{h})$, for every $\bar{h} \in H$. We will use $(f \mid h)$ to denote $\left(f_{1}\left|h, \ldots, f_{n}\right| h\right)$ for every $f \in S$ and $h \in H$.

Any strategy $f \in F$ induces an outcome $\pi(f)$ as follows:

$$
\pi^{1}(f)=f(e) \quad \pi^{k}(f)=f\left(\pi^{1}(f), \ldots, \pi^{k-1}(f)\right),
$$

for $k \in \mathbb{N}$. Thus, we have define a function $\pi: F \rightarrow S^{\infty}$, where $S^{\infty}=$ $S \times S \times \cdots$.

Let $M \geq 0$ be such that $\left|u_{i}(s)\right| \leq M$, for all $s \in S$, and $i \in N$. Then, any outcome $\pi \in S^{\infty}$ induces two elements in $l^{\infty}$, one for each player, as follows

$$
x_{i}^{k}(\pi)=u_{i}\left(\pi^{k}\right)
$$

for all $k \in \mathbb{N}$. Thus, we have define a function $x_{i}: S^{\infty} \rightarrow l^{\infty}$, for all $i=1,2$. 
For $x, y \in l^{\infty}, x \geqq y$, means $x_{k} \geq y_{k}$, for all $k \in \mathbb{N} ; x \geq y$ means $x \neq y$ and $x \geqq y$.

Let for each $i=1,2$, and $k \in \mathbb{N}, U_{i}^{k}: l^{\infty} \rightarrow \mathbb{R}$ be given. The payoff for player $i, i=1,2$, from his point of view in period $k \in \mathbb{N}$ of a strategy $f \in F$ in the supergame of $G$ is defined to be $U_{i}^{k}\left(x_{i} \circ \pi(f)\right)$.

A strategy vector $f \in F$ is a subgame perfect equilibrium of the supergame of $G$ if $U_{i}^{k}\left(x_{i}(h \cdot \pi(f \mid h))\right) \geq U_{i}^{k}\left(x_{i}\left(h \cdot \pi\left(g_{i}, f_{-i} \mid h\right)\right)\right)$, for all $i=1,2, k \in \mathbb{N}$, $h \in H_{k-1}$ and $g_{i} \in F_{i}$. Let $E \Pi$ denote the set of subgame perfect equilibrium outcomes.

\section{Equilibrium outcomes}

In this section we state and prove our main result.

Theorem 1 Suppose that for all $k \in \mathbb{N}$, and $i=1,2$,

1. $U_{1}^{k}\left(x_{1}(\pi)\right)+U_{2}^{k}\left(x_{2}(\pi)\right)=0$, for all $\pi \in S^{\infty}$,

2. $U_{i}^{k}$ is strictly increasing: $x, y \in l^{\infty}$ and $x \geq y$ implies $U_{i}^{k}(x)>U_{i}^{k}(y)$.

Then, $E \Pi=N E^{\infty}$ and $u_{i}\left(\pi^{k}\right)=v_{i}$ for all $\pi \in E \Pi, i=1,2$, and $k \in \mathbb{N}$.

Proof. Let $\pi \in E \Pi, i=1,2$, and $k \in \mathbb{N}$. By 2 ,

$$
\begin{aligned}
& U_{i}^{k}\left(x_{i}(\pi)\right) \geq U_{i}^{k}\left(x_{i}^{1}(\pi), \ldots, x_{i}^{k-1}(\pi), \max _{s_{i}} u_{i}\left(s_{i}, \pi_{-i}^{k}\right), v_{i}, v_{i} \ldots\right) \geq \\
& \geq U_{i}^{k}\left(x_{i}^{1}(\pi), \ldots, x_{i}^{k-1}(\pi), v_{i}, v_{i}, \ldots\right):=\bar{v}_{i}^{k} .
\end{aligned}
$$

Let $\alpha$ be a Nash equilibrium of $G$; thus, in particular, $u_{i}(\alpha)=v_{i}$. By letting $\tilde{\pi}=\left(\pi^{1}, \ldots, \pi^{k-1}, \alpha, \alpha, \ldots\right)$, we conclude by 1 that $\bar{v}_{1}^{k}+\bar{v}_{2}^{k}=$ $U_{1}^{k}\left(x_{1}(\tilde{\pi})\right)+U_{2}^{k}\left(x_{2}(\tilde{\pi})\right)=0$. Also, by $1, U_{1}^{k}\left(x_{1}(\pi)\right)+U_{2}^{k}\left(x_{2}(\pi)\right)=0$. Hence, $U_{k}\left(x_{i}\right)=\bar{v}_{i}^{k}$.

We therefore conclude that,

$$
\begin{aligned}
& U_{k}\left(x_{i}^{1}(\pi), \ldots, x_{i}^{k-1}(\pi), \max _{s_{i}} u_{i}\left(s_{i}, \pi_{-i}^{k}\right), v_{i}, v_{i} \ldots\right)= \\
& =U_{k}\left(x_{i}^{1}(\pi), \ldots, x_{i}^{k-1}(\pi), v_{i}, v_{i}, \ldots\right),
\end{aligned}
$$

and so by $2, \max _{s_{i}} u_{i}\left(s_{i}, \pi_{-i}^{k}\right)=v_{i}$. 
Since $u_{i}\left(\pi^{k}\right)=x_{i}^{k}(\pi) \leq \max _{s_{i}} u_{i}\left(s_{i}, \pi_{-i}^{k}\right)=v_{i}$, for all $k \in \mathbb{N}$, and $U_{i}^{1}\left(x_{i}(\pi)\right) \geq U_{i}^{1}\left(v_{i}, v_{i}, \ldots\right)$, it follows that

$$
u_{i}\left(\pi^{k}\right)=v_{i}=\max _{s_{i}} u_{i}\left(s_{i}, \pi_{-i}^{k}\right) ;
$$

hence, $\pi^{k}$ is a Nash equilibrium.

Corollary 1 Suppose that for all $k \in \mathbb{N}$

1. $U_{i}^{k}=U_{k}$, for $i=1,2$,

2. $U_{k}$ is additive: $U_{k}(x+y)=U_{k}(x)+U_{k}(y)$, for all $x, y \in l^{\infty}$,

3. $U_{k}$ is strictly increasing: $x, y \in l^{\infty}$ and $x \geq y$ implies $U_{k}(x)>U_{k}(y)$.

Then, $E \Pi=N E^{\infty}$ and $u_{i}\left(\pi^{k}\right)=v_{i}$ for all $\pi \in E \Pi, i \in N$, and $k \in \mathbb{N}$.

The following example shows that we cannot dispense with additivity. The stage game is the matching pennies:

\begin{tabular}{|c|c|c|}
\hline $1 \backslash 2$ & $H$ & $T$ \\
\hline$H$ & $1,-1$ & $-1,1$ \\
\hline$T$ & $-1,1$ & $1,-1$ \\
\hline
\end{tabular}

Assume time-consistency, and let $w=(-1,1,-1,1, \ldots)$.

The preferences are, for $\delta \in(0,1)$ and $M>0$,

$$
U(x)=\left\{\begin{array}{l}
(1-\delta) \sum_{k=1}^{\infty} \delta^{k-1} x_{k}+M \quad \text { if } \quad x \geqq w \\
(1-\delta) \sum_{k=1}^{\infty} \delta^{k-1} x_{k} \quad \text { otherwise. }
\end{array}\right.
$$

This preferences are strictly increasing because $\geqq$ is transitive and the discounted sum is strictly increasing. Define $\pi$ as follows $\pi^{1}=(H, H), \pi^{2}=$ $(T, H), \pi^{3}=(H, H), \pi^{4}=(T, H), \ldots$, and define

$$
f_{i}(h)=\left\{\begin{array}{l}
\pi_{i}^{k} \quad \text { if } h=\left(\pi^{1}, \ldots, \pi^{k-1}\right) \\
\text { play H with } 1 / 2 \text { probability otherwise. }
\end{array}\right.
$$

Then $f$ is a subgame perfect equilibrium and $\pi$ is a SPE outcome (and it doesn't consist of Nash equilibria of the stage game). This is so because the 
payoff of the equilibrium path is $M-\frac{(1-\delta)^{2}}{1-\delta^{2}}$, and the payoff from deviating is 1 . By choosing $M$ big enough, we can deter deviations.

Let for $h \in H$ and $r \in \mathbb{N}, \lambda^{r} h:=\left(h^{1}, \ldots, h^{r}\right)$ and $\mu^{r} h:=\left(h^{r}, h^{r+1}, \ldots\right)$. We say that $U_{k}, k \in \mathbb{N}$, is independence of the past if for all $x, y \in S^{\infty}$ satisfying $\lambda^{k-1} x=\lambda^{k-1} y$ then $U_{k}(x) \geq U_{k}(y)$ if and only if $U_{k}\left(\mu^{k} x\right) \geq$ $U_{k}\left(\mu^{k} y\right)$. If, for all $k \in \mathbb{N}, U_{k}$ is independent of the past, then $f \in F$ is a subgame perfect equilibrium if and only if $U_{k}\left(x_{i} \circ \pi(f \mid h)\right) \geq U_{k}\left(x_{i} \circ\right.$ $\left.\pi\left(g_{i}, f_{-i} \mid h\right)\right)$, for all $i=1,2, k \in \mathbb{N}, h \in H_{k-1}$ and $g_{i} \in F_{i}$.

Theorem 2 Suppose that for all $k \in \mathbb{N}$

1. $U_{i}^{k}=U_{k}$, for $i=1,2$,

2. $U_{k}$ is independent of the past,

3. $U_{k}(x+y) \geq U_{k}(x)+U_{k}(y)$, for all $x, y \in l^{\infty}$,

4. $U_{k}(\alpha, \alpha, \ldots)=\alpha$, for all $\alpha \in \mathbb{R}$,

5. $U_{k}$ is strictly increasing: $x, y \in l^{\infty}$ and $x \geq y$ implies $U_{k}(x)>U_{k}(y)$.

Then, $E \Pi=N E^{\infty}$ and $u_{i}\left(\pi^{k}\right)=v_{i}$ for all $\pi \in E \Pi, i=1,2$, and $k \in \mathbb{N}$.

Proof. Let $\pi \in E \Pi, i=1,2$, and $k \in \mathbb{N}$. For $t \geq k$, let $x_{i}^{t}=u_{i}\left(\pi^{t}\right)$, and $x_{i}=\left(x_{i}^{k}, x_{i}^{k+1}, \ldots\right)$. By 3 , and 4 ,

$$
U_{k}\left(x_{i}\right) \geq U_{k}\left(\max _{s_{i}} u_{i}\left(s_{i}, \pi_{-i}^{k}\right), v_{i}, v_{i} \ldots\right) \geq U_{k}\left(v_{i}, v_{i}, \ldots\right)=v_{i} .
$$

By 2, and 3,

$$
U_{k}\left(x_{1}\right)+U_{k}\left(x_{2}\right) \leq U_{k}\left(x_{1}+x_{2}\right)=0 .
$$

Because $v_{1}+v_{2}=0$, it follows that

$$
U_{k}\left(x_{i}\right)=v_{i}
$$

Thus, by $4, \max _{s_{i}} u_{i}\left(s_{i}, \pi_{-i}^{k}\right)=v_{i}$. Since $u_{i}\left(\pi^{k}\right)=x_{i}^{k} \leq \max _{s_{i}} u_{i}\left(s_{i}, \pi_{-i}^{k}\right)=$ $v_{i}$, then $u_{i}\left(\pi^{k}\right)=v_{i}$. Since this equality holds for all $k \in \mathbb{N}$, it follows that $x_{i}=\left(v_{i}, v_{i}, \ldots\right)$, and so by 4 ,

$$
u_{i}\left(\pi^{k}\right)=v_{i}=\max _{s_{i}} u_{i}\left(s_{i}, \pi_{-i}^{k}\right) ;
$$

hence, $\pi^{k}$ is a Nash equilibrium. 


\section{References}

[1] Goldman, S., (1980), "Consistent Plans", Review of Economic Studies, 47, 533-537.

[2] Jovanovic, B., and D. Stolyarov, (2000), "Ignorance is Bliss," Manuscript, New York University.

[3] Kocherlakota, N., (2001), "Looking for Evidence of Time-Inconsistent Preferences in Asset Market Data," Federal Reserve Bank of Minneapolis Quarterly Review, v. 25, no. 3, 13-24.

[4] Laibson, D., (1997), "Golden Eggs and Hyperbolic Discounting," Quarterly Journal of Economics, 112, 443-477.

[5] Peleg, B., and M. Yaari (1973), "On the Existence of a Consistent Course of Action when Tastes are Changing," Review of Economic Studies, 40, $1-24$.

[6] Strotz, R. (1955), "Myopia and Inconsistency in Dynamic Utility Maximization," Review of Economic Studies, 23, 165-180. 